\title{
Avances en la descripción morfosintáctica de la transitividad en el asháninka del alto Perené ${ }^{1}$
}

\author{
Advances in the morphosyntactic description of \\ transitivity in the Asháninka of the upper Perené
}

\author{
Alicia Alonzo Sutta \\ Universidad Nacional Mayor de San Marcos, Lima, Perú \\ https://orcid.org/oooo-0002-9063-508X \\ aalonzos@unmsm.edu.pe
}

\begin{abstract}
Resumen
La visión tradicional acerca de la transitividad se basa en que la lengua presenta verbos transitivos e intransitivos. Sobre esta base, en las cláusulas transitivas se produce una acción de transferencia de un sujeto a un objeto. De acuerdo con esta afirmación, el verbo transitivo es el que denota sucesos acerca del objeto, sea una acción sobre él o un cambio de estado de este. Sin embargo, los estudios posteriores a dicha perspectiva, realizados por Hopper y Thompson (1980) discrepan de lo anterior y proponen que la transitividad no responde a una simple transferencia del sujeto hacia el objeto. Para los autores, la transitividad es una propiedad de las lenguas que se caracteriza por el cumplimiento de determinados parámetros, que indican el mayor o menor grado de transitividad de las cláusulas dentro de un contexto discursivo. El presente estudio tiene como fin describir las características morfosintácticas que muestran un mayor grado de transitividad en las cláusulas del asháninka del alto Perené.
\end{abstract}

Palabras clave: transitividad, asháninka, verbos, morfemas, objeto

\begin{abstract}
The traditional view of transitivity is based on the fact that the language has both transitive and intransitive verbs. On this basis, in transitive clauses there is an action of transference from a subject to an object. According to this statement, the transitive verb is the one that denotes events about the object, either an action on it or a change of state of it. However, studies subsequent to this perspective, carried out by Hopper and Thompson (1980) disagree with the above and propose that transitivity does not respond to a simple transference from the subject to the object. For the authors, transitivity is a property of languages characterized by the fulfillment of
\end{abstract}

1 El estudio que presentamos en este artículo corresponde a una parte de la tesis de maestría titulada «Análisis morfosintáctico de la transitividad en oraciones de un relato de la lengua asháninka», la cual fue auspiciada por el VRIP (Vicerrectorado de Investigación y Posgrado) de la unMsm a través del Programa de Promoción de Tesis de Posgrado para Docentes de la Universidad en el año 2019. 
Avances en la descripción morfosintáctica de la transitividad en el asháninka...

Alicia Alonzo Sutta

certain parameters, which indicate the greater or lesser degree of transitivity of clauses within a discursive context. The present study aims to describe the morphosyntactic characteristics that show a higher degree of transitivity in the clauses of Upper Perene Asháninka.

Key words: transitivity, asháninka, verbs, morphemes, object

FRecibido: I8-05-202I

Aprobado: 15-06-202I

Publicado: I9-I2-202I

\section{Introducción}

Una de las lenguas que forma parte del diverso panorama lingüístico en la Amazonía es el asháninka, lengua perteneciente a la familia lingüística Arawak que ocupa fundamentalmente siete departamentos del Perú, entre los que se cuentan, Ayacucho, Cusco, Loreto, Ucayali, Huánuco, Pasco y principalmente Junín. De acuerdo con las fuentes oficiales, es el grupo social indígena con mayor número de hablantes (Minedu, 2018).

Desde mediados del siglo pasado, la lengua asháninka, llamó la atención de estudiosos, como Dirks (1953), Kindberg (1975), Kindberg (1980), Heitzman (1975), Payne, D, Payne J. y Sánchez (1982), García (1997), quienes describieron gramaticalmente las variantes de esta lengua. Dialectos que fueron denominadas «campa», «campa ashaninca», «asháninca» $y$ «ashéninca». Actualmente denominadas lenguas asháninka y ashéninka²

Las temáticas abordadas en mayor proporción fueron de orden fonético-fonológico y léxico-semántico. En el primer caso, se estudiaron los componentes fonético-fonológicos de la lengua a fin de describir los sonidos y fonemas que constituyen el sistema de esta lengua. En el segundo, muy productivo, por cierto, se registraron las unidades léxicas que constituyen el vocabulario de las variantes dialectales de la lengua asháninka. Hasta la actualidad se cuenta con más de cinco diccionarios y vocabularios correspondientes a distintas variedades, los cuales fueron elaborados por Payne (2008), Kindberg (2008), Minedu (2000), Payne (1989), Cushimariano y Sebastián (2008), MED y Jacinto (2017), Jacinto y Bernales (2007), Mihas (2014), entre otros.

En relación con el componente morfosintáctico, los estudios han priorizado la identificación y registro de morfemas para establecer la estructura morfosintáctica de la palabra nominal y verbal. Los estudios gramaticales fueron realizados

2 El nombre ashéninka se registra oficialmente desde el año 2017 para denominar a la lengua del pueblo del mismo nombre, la cual mantiene una estrecha relación con el pueblo asháninka en términos históricos, sociales, políticos, culturales y lingüísticos. 
por Kindberg (1975) en la variedad denominada «asháninca» correspondiente a la variedad empleada en los ríos Ene y Tambo fundamentalmente. La descripción gramatical en la variedad del Apurucayali fue realizada por Payne, Payne y Sánchez (2008), otras descripciones morfosintácticas bastante breves en la variedad ucayalina y del Pichis fueron realizadas por García (1997) y García (2007), respectivamente.

El presente estudio tiene como fin describir ciertas características morfosintácticas de la transitividad en el asháninka del alto Perené, lengua empleada en Chanchamayo (Junín). Para tal fin se aplicarán a las cláusulas tres parámetros propuestos por Hopper y Thompson (1980) en la conocida hipótesis de la transitividad. La propuesta implica que los parámetros caracterizan a las cláusulas como más o menos transitivas. A un mayor número de rasgos presentes en la cláusula, se alcanza una escala más alta de transitividad. Los rasgos evidenciados en asháninka corresponden al número de participantes, la telicidad y la afirmación.

\section{Antecedentes}

La revisión de literatura acerca de la transitividad nos llevó a hallar estudios acerca de descripciones gramaticales de distintas lenguas amerindias, especialmente las de la familia Arawak. Vale indicar que por el propósito que tuvieron estas investigaciones, en su mayoría, se orientaron fundamentalmente a dar cuenta de las características morfosintácticas generales de las lenguas, aspectos muy relevantes e imprescindibles para todo estudio gramatical. Dentro de dichas descripciones se abordaron, de una manera bastante inicial, el estudio de los verbos y en menor medida el de la transitividad.

Los estudios gramaticales sobre el verbo como unidad principal de la cláusula son recientes y parciales, datan de mediados del siglo pasado. Son pocos los que se han aproximado al estudio del verbo y/o del predicado en estas lenguas; quizás por la alta complejidad que encierra esta unidad lingüística, por los procesos morfofonológicos que en él ocurren, entre otros aspectos de carácter morfológico, sintáctico y semántico.

Los primeros estudios descriptivos en esta lengua amerindia se realizaron a mediados del siglo xx por parte de los investigadores del ILv. Una de las descripciones lingüísticas preliminares se halla en Campa (Arawak) phonemes (1953); en este estudio, Sylvester Dirks presenta una muy breve descripción fonético-fonológica de la lengua. Junto a estos aspectos, también realiza una breve descripción 
Avances en la descripción morfosintáctica de la transitividad en el asháninka... Alicia Alonzo Sutta

morfológica acerca de la estructura del nombre, la conformación de la palabra nominal y la configuración de la palabra verbal. Vale indicar que aun cuando el estudio es bastante sucinto, provee, desde una perspectiva morfológica y sintáctica, una información preliminar que sirvió de fuente para posteriores investigaciones gramaticales.

En el estudio Los morfemas verbales del campa ashaninca, Kindberg (1975) expone en orden paradigmático un conjunto de morfemas gramaticales de tipo prefijal y sufijal que se asignan tanto a nombres como a verbos; es decir, afijos nominales y verbales contextualizados en frases específicas. Dentro del conjunto de afijos verbales, cabe destacar un limitado número de prefijos pronominales que se adjuntan a las raíces verbales. Complementariamente, el autor registra un alto número de sufijos verbales que ascienden aproximadamente a ochenta, algunos de ellos con alternancias alomórficas. Vale indicar que, el autor, incluye estos morfemas en cláusulas breves que brindan información valiosa. A pesar de que el estudio no corresponde a una descripción gramatical, sino a un paradigma de afijos incluidos en frases y cláusulas breves, permite reconocer importantes características gramaticales de la variedad que describe.

Posteriormente, en el estudio Formas vacías en el campa ashaninca, Kindberg (1975), trata un fenómeno morfofonológico que ocurre en la construcción de la palabra verbal de esta variedad. El autor advierte que en el proceso de inflexión del verbo tiene lugar el proceso de inserción de segmentos «t» $\mathrm{y}$ «a»; este fenómeno, sostiene el autor, únicamente se produce en contextos exclusivamente verbales y tales elementos no corresponden a ningún morfema verbal; sin embargo, se presentan en forma reiterada en la configuración del verbo por lo que él denominará «formas vacías». Con los estudios posteriores, hoy se sabe que estos segmentos forman parte del proceso epentético que ocurre en el verbo de todas las variedades de la lengua asháninka.

En el marco de los estudios tipológicos y siguiendo los universales propuestos por Greenberg (1963), tiene lugar el estudio denominado Morfología, fonología y fonética del ashéninca del Apurucayali (Campa Arawak Preandino) realizado por David Payne, Judith Payne y Jorge Sánchez (2008). Dentro del componente morfosintáctico de esta lengua, los autores, destacan la capacidad del verbo de admitir más morfemas que cualquier otro tipo de palabra. Por su carácter aglutinativo, al verbo se adjuntan morfemas flexivos y derivativos. Respecto al tratamiento que recibe el verbo dentro de la morfología verbal, sostienen que los temas verbales precisan de subcategorizaciones, las cuales indican restricciones de ocurrencia 
entre el tema verbal y los sufijos que constituyen las marcas del complemento directo e indirecto. Respecto de la transitividad, los autores indican que el ashéninca del Apurucayali presenta temas verbales intransitivos que son susceptibles de llevar el prefijo de persona que constituye la marca de sujeto. Asimismo, presenta temas verbales transitivos que son susceptibles de admitir sufijos que indican la persona del complemento sean estos indirecto y/o directo.

La pronominalización, o sea, los pronombres expresados como morfemas gramaticales en los verbos constituyen una característica del verbo, y son estos los que asumen la función de sujeto o agente en la oración. Por ejemplo (Payne, 2008, p. 22):
(1) olga o-čhik-ak-i-ri
kitairiki
Olga 3f-cortar-PFV-NF-3
sajino
Olga cortó el sajino.

En el verbo č $i k$ - 'cortar' se encuentran el prefijo $o$ - correspondiente al pronombre de $3^{\circ}$ persona no masculino y también se adjunta la marca sufijal ri- 'sajino' que corresponde al complemento directo de dicho verbo. Complementariamente, a lo indicado, el autor sostiene que el verbo, además, presenta categorías de persona, número, tiempo, modo y género.

Posteriormente en Lecciones para el aprendizaje del idioma asheninca, Judith Payne (2008, p. 129), afirma que los sufijos llamados de complemento están pospuestos al sufijo de tiempo en el verbo y, por lo general, se encuentran al final de la palabra verbal. También sostiene que los sufijos $-n a$ y $-m i$ refieren a las personas gramatical 'me', 'a mí' y 'te', 'a ti' respectivamente; sin embargo, los sufijos -ri y -ro además de referirse a personas, -ri se refiere a elementos animados y -ro a elementos inanimados, aunque con ciertas excepciones. A continuación, presentamos los sufijos denominados por la autora como sufijos de complemento y añade que un mismo sufijo puede representar al complemento directo e indirecto. 
Avances en la descripción morfosintáctica de la transitividad en el asháninka... Alicia Alonzo Sutta

\section{Cuadro 1. Sufijo de complemento}

\begin{tabular}{|ll|}
\hline - na & 'me' \\
- mi & 'te' \\
- ri & 'lo, 'le' (masculino) \\
- ro & 'la', 'lo' (neut.), 'le' (f.) \\
- ai & 'nos' \\
\hline
\end{tabular}

Nota: Tomado de Payne (1987)

Por la naturaleza del texto orientado a la enseñanza, la autora presenta los morfemas gramaticales (sufijos) que constituyen complementos de ciertos verbos; sin embargo, no hace referencia, en forma explícita, a los verbos transitivos.

Posteriormente, en Generalidades de la morfología y fonología del asheninca del Ucayali, sobre la variante empleada en la región del alto Ucayali, Gabriela García (1997) registra los morfemas que se adjuntan a los diferentes tipos de verbos de esta variante. Cabe indicar que este documento se caracteriza por ser bastante breve; sin embargo, los datos morfosintácticos aportan en el reconocimiento de la estructura de la palabra verbal y sobre todo de las cláusulas cuyos verbos presentan sufijos -ri y -ro, los que corresponden a la marca del complemento del verbo.

Ejemplo:

(2) maria o-ñaak-e-ri konoya ha-a-ak-e-ri huan

María 3f-ver-PFV-NF-3F motelo 3m-cazar-PFV-NF-rel juan

'María vio el motelo que cazó Juan'

En Fonología del asháninka del río Pichis, García, F. (2007), investigador de FORMABIAP, expone un estudio bastante breve que realiza en la cuenca del río Pichis. Los datos sobre aspectos fonético-fonológicos son básicos En este estudio, se confirma la morfología nominal y verbal que presenta la lengua asháninka hablada en el Pichis (Pasco). Aunque la data que se registra es bastante escueta, sin embargo, permite confirmar la manera en que los verbos se han configurado. Sostiene que de acuerdo con la estructura morfológica en asháninka del Pichis existen dos tipos de palabras: una verbal y otra nominal (2007, p. 50). Respecto de la primera, sostiene que un verbo transitivo está conformado de un prefijo de índice actancial 1, seguido de una raíz verbal, al que se añade el sufijo aspectual y finalmente el índice actancial 2 del participante.

274 Lengua y Sociedad. Revista de Lingüística Teórica y Aplicada 
Avances en la descripción morfosintáctica de la transitividad en el asháninka...

Alicia Alonzo Sutta

Por ejemplo: García (2007, pp. 50-51):

(3) [ikantiro]

$\mathrm{i}+\mathrm{kant}+\mathrm{i}+\mathrm{ro}$

3M-decir-FAC-3F

'él dijo a ella'

(4) [ikantaheitakiri]

$\mathrm{i}+\mathrm{kant}+\mathrm{a}+\mathrm{hei}+\mathrm{t}+\mathrm{ak}+\mathrm{i}+\mathrm{ri}$

3M-decir-PL-PFV-FAC-3M

'él les dijo a ellos'

(5) [jamenaßetaro]

$\mathrm{j}+\mathrm{amen}+\mathrm{a}+\beta \mathrm{e}+\mathrm{t}+\mathrm{a}+\mathrm{ro}$

3M-mirar-ENF-voz-3F

'él miró a ella'

A pesar de que el estudio en mención no aborda el fenómeno de la transitividad, este sí da cuenta de la estructura verbal y de los argumentos que contiene el verbo expresado mediante los sufijos -ro y -ri. En palabras del autor, al índice actancial 2, le corresponde el segundo participante de la oración.

El reciente estudio Essentials of Ashéninka Perené Grammar realizado por Mihas (2010) en la variedad del Alto Perené da cuenta del componente fonético-fonológico y del morfosintáctico. En este último se enfatiza la aglutinación de morfemas y del patrón polisintético de la lengua; asimismo se reconocen las clases de palabras de esta lengua; la serie de afijos que corresponden a cada cual; el orden específico que presentan; la mayor o menor distancia que mantienen con relación al núcleo lexical; en lo que respecta a los verbos transitivos, la autora sostiene lo siguiente:

Asheninka Perené verbs divide into transitivity classes depending on the transitivity type of clauses they may appear in. Two basic transitivity clause types include intransitive clauses which have one obligatory argument in S (intransitive subject) function and transitive clauses which have two arguments in A (transitive subject) and $\mathrm{O}$ (transitive object) functions (Dixon 2010 a: 115-6). The core arguments may be expressed by the person clitics, personal pronouns, and demostrative or noun referents. Examples of verb 
Avances en la descripción morfosintáctica de la transitividad en el asháninka...

Alicia Alonzo Sutta

roots expressing intransitivity values are kam 'die', shiy 'run'; kamaraNk 'vomit'; transitive verb roots are am 'bring', a 'take', pos 'hit. (Mihas, 2010, p. 135)

De acuerdo con criterios morfosintácticos, la autora sostiene que en la variedad del Perené los verbos como am 'bring', a 'take', pos 'hit' son los encargados de expresar transitividad y; que los elementos sintácticos requeridos en una cláusula transitiva son los argumentos centrales agente $(A)$ y objeto $(\mathrm{O})$ expresados mediante referentes nominales como clíticos de persona, pronombres personales y demostrativos. Asimismo, las cláusulas intransitivas necesariamente cuentan con un argumento obligatorio, expresado como $S$, que corresponde al sujeto intransitivo.

Respecto a la morfología verbal, los autores arriba mencionados sostienen que la lengua asháninka es predominantemente sufijante y con un número limitado de prefijos. Respecto de ello, vale indicar que las marcas prefijales en los verbos $\mathrm{y}$ en los nombres son las mismas e incluso son consideradas como homófonas por Lawrence (2013, p. 24) dado que son idénticas en su estructura. Cuando estos prefijos se adjuntan al nombre constituyen la marca del poseedor; $y$, cuando se adjuntan al verbo, cumplen el rol de sujeto. Esta característica morfosintáctica se evidencia en todos los dialectos de la lengua asháninka y en lenguas estrechamente relacionadas a ella como matsigenga y Kakinte.

Ejemplos:

Asháninka del Alto Perené

Sustantivo: Alonzo (2015)

(6) Antaro No-banko-ki

Grande 1PSs-casa-LOC

'En mi casa grande'

Ashéninca del Apurucayali

Verbo: Payne et al. (2008, p. 31)

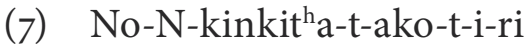

1S-FUT-contar cuento-EP-DAT-EP-FUT-ABSL

'Voy a contar acerca de él'

276 Lengua y Sociedad. Revista de Lingüística Teórica y Aplicada 
Avances en la descripción morfosintáctica de la transitividad en el asháninka...

Alicia Alonzo Sutta

Matsigenka

Sustantivo: Snell (2011, p. 298)

(8) Pi-nevatyage-te

1PSS-sobrina-PSS

'Mi sobrina'

Verbo: Lorenzo (2010)

(9) Pi-timporoka-guito-t-ake-na

2-romper-cabeza-EP-Perf-сомP1P

'Me has roto la cabeza'

Kakinte

Sustantivo: Castillo (2017, p. 73)

(10) No-motiha

1PSs- barriga

'Mi barriga'

Verbo: Swift (2008, p. 199)

(11) No-tasonk-ak-e

1 S- soplar-PFV-NO FUT.

'Yo soplé'

En los datos citados, se confirma que los prefijos referenciales de persona no- y $p i$ - antepuestos al nombre y verbo coinciden en su estructura. Cuando se encuentran frente al nombre, se establece una relación de dependencia, es decir, funge de poseedor, y cuando se adjunta al verbo, asume el rol de sujeto o agente.

Por otro lado, en Dixon y Aikhenvald (2000, p. 146), Aikhenvald estudia tariana, lengua Arawak, hablada en Brasil; ella sostiene que la gramática de esta lengua presenta distintos tipos de verbos. Los afijos que se añaden al verbo, por lo general, aparecen pospuestos a él; por ello considera que tariana presenta una compleja estructura verbal. La autora sostiene que, en casi todas las lenguas, los verbos son estrictamente transitivos e intransitivos y en ciertas lenguas pueden tener el valor de transitividad. Específicamente, en dicha lengua, los verbos transitivos tienen al 
Avances en la descripción morfosintáctica de la transitividad en el asháninka...

Alicia Alonzo Sutta

A (agente) como equivalente al S (sujeto), esto significa que la frase nominal (O) objeto puede ser opcionalmente omitida, tal como se comprueba en la siguiente oración:

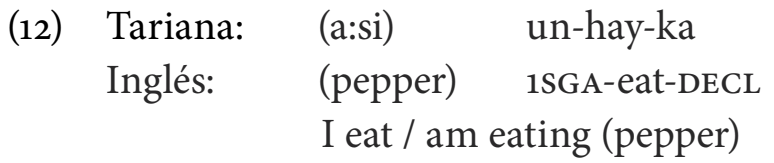

Varios estudiosos ya habían señalado que el proceso de formación de la palabra verbal, en lenguas Arawak, es un asunto bastante complejo. Un proceso bastante común en la formación del verbo es la afijación; en lo que se refiere solo a los prefijos, se encuentran los pronombres personales, el morfema que expresa el estado irrealis y un prefijo causativo. En la sufijación se va a notar una gran diversidad y complejidad ya que la mayoría de los sufijos flexivos y derivativos presentan una ubicación determinada en la palabra verbal, sostiene Dixon y Aikhenvald (1999, p. 65).

Considerando esto, el verbo es el que presenta diferentes categorías como el tiempo, aspecto, modo, causatividad, reciprocidad, entre otros. Una marca verbal relevante y obligatoria es la que indica la diferencia entre eventos realis/irrealis; los eventos o sucesos con marca realis son los que ocurrieron en el pasado o que están sucediendo, frente a hechos, sucesos o eventos aún no realizados tendrán la marca irrealis.

Como parte de la clase de los verbos activos, se encuentran los verbos transitivos que se caracterizan por presentar dos argumentos nucleares, el agente y objeto, representado por A y O respectivamente; asimismo, los verbos intransitivos, que están constituidos de un núcleo argumental, asumen la función de sujeto (S).

En el estudio reciente Morfología en lenguas Arawak, Aikhenvald (2020, p. 20) registra datos gramaticales de distintas lenguas Arawak de Sudamérica; en lo que respecta al ashéninka del Perené, sostiene que esta no presenta marcas de caso, por lo que se confirma que no es una lengua de caso a pesar de que presenta una única marca de caso: locativo $-k i$.

A través de los distintos estudios presentados anteriormente, se comprueba que se considera que existen cláusulas con verbos transitivos e intransitivos, donde los primeros estarán constituidos de dos participantes relacionados por la acción transferida del primero hacía el segundo. Desde una perspectiva morfosintáctica,

278 Lengua y Sociedad. Revista de Lingüística Teórica y Aplicada 
Avances en la descripción morfosintáctica de la transitividad en el asháninka...

Alicia Alonzo Sutta

los temas verbales en asháninka son considerados transitivos y estos son pasibles de admitir sufijos que marcan el complemento directo.

\section{Marco conceptual del estudio}

Una caracterización tradicional acerca de la transitividad considera que una lengua cuenta con verbos transitivos y verbos intransitivos. Esta primera propuesta presenta un problema respecto de una división de los verbos dentro de una lengua, clasificándolos en dos grupos claramente separados; entendiéndose que hay un corte o división entre ambas clases de verbos.

La segunda caracterización tradicional afirma que la cláusula transitiva presenta un objeto, y este corresponde al segundo participante. En la cláusula hay una acción que se transfiere desde el sujeto hacia el objeto.

Estas dos caracterizaciones se emplearon por mucho tiempo en las descripciones gramaticales, sin embargo, en la segunda mitad del siglo xx con el incremento de las descripciones gramaticales en lenguas no indoeuropeas principalmente, se llegó a desarrollar nuevos planteamientos acerca de la transitividad.

Bajo este contexto, surge la hipótesis de la transitividad propuesta por Hopper y Thompson (1980), la cual supera el concepto tradicional basado en la división de verbo transitivo e intransitivo y afirma que, en lugar de este corte o división, se debe considerar que entre dichos verbos existe un continuum. Asimismo, se supera la noción de que la transitividad se halla en los verbos únicamente, por el contrario, esta constituye una propiedad que no se encuentra en el verbo, sino en toda la cláusula; puesto que es en ella, donde, además de considerar aspectos estructurales de tipo morfosintáctico, se deben considerar aspectos semánticos que únicamente se distinguen en la cláusula en su conjunto. En suma, la transitividad como propiedad de la cláusula es un hecho gradual que depende de los rasgos que estén presentes para indicar que ella alcanza un mayor o menor grado de transitividad.

En vista de que el fenómeno de la transitividad constituye una propiedad de las cláusulas de una lengua determinada, en ellas se debe dar cumplimiento a determinados parámetros. Los parámetros o características propuestas por los autores mencionados son diez y abarcan aspectos de tipo morfosintáctico y semántico-discursivos. 
Avances en la descripción morfosintáctica de la transitividad en el asháninka...

Alicia Alonzo Sutta

El cumplimiento de un mayor número de parámetros en la cláusula indicará que ella alcanza un alto grado de transitividad y viceversa, aquella cláusula con menos parámetros tendrá un menor grado de transitividad.

A continuación, presentamos los parámetros de la transitividad según Hopper y Thompson (1980):

Cuadro 2. Transitividad

\begin{tabular}{|c|c|c|c|}
\hline \multicolumn{4}{|c|}{ Parameters of transitivity } \\
\hline & & High & Low \\
\hline A. & Participants & 2 or more participants, $\mathrm{A}$ y O & 1 participant \\
\hline B. & Kinesis & Action & Non-action \\
\hline c. & Aspect & Telic & Atelic \\
\hline D. & Punctuality & Punctual & Non-Punctual \\
\hline E. & Volitionality & Volitional & Non-Volitional \\
\hline F. & Affirmation & Affirmative & Negative \\
\hline G. & Mode & Realis & Irrealis \\
\hline $\mathrm{H}$. & Agency & A high in potency & A low in potency \\
\hline I. & Affectedness of $\mathrm{O}$ & O totally affected & O not affected \\
\hline $\mathrm{J}$. & Individuation of $\mathrm{O}$ & O highly individuated & O not-individuated \\
\hline
\end{tabular}

Nota: Tomado de Hopper y Thompson (1980)

Para el presente estudio, seleccionamos los parámetros que guardan una relación directa con aspectos morfosintácticos de la lengua; en esa medida, los rasgos seleccionados para determinar los grados de transitividad harán referencia al número de participantes en la oración, la telicidad que indique una acción concluida y la afirmación de la cláusula en la que no existan marcas de negación.

\section{Metodología}

La presente investigación es de tipo descriptivo-explicativo. Se considera descriptiva porque permite identificar ciertas características gramaticales que presentan las cláusulas en la lengua asháninka del alto Perené. En ese sentido, se identificarán las unidades principales que presentan las cláusulas (verbos y argumentos) así como la conformación morfosintáctica de las cláusulas que presentan transitividad. 
Avances en la descripción morfosintáctica de la transitividad en el asháninka...

Alicia Alonzo Sutta

\subsection{Población}

La elección del área geográfico-espacial es de suma importancia en esta investigación, en particular en la lengua asháninka que cuenta con una alta variabilidad dialectal en la región. Después de conocer ciertas áreas dialectales, se decidió realizar el presente estudio en la cuenca del alto Perené, puesto que la variante empleada en esta región ha sido considerada por la Unesco como una lengua cuya vitalidad se encuentra seriamente en peligro (Minedu, 2013, p. 54) debido al desplazamiento de esta por parte del castellano.

Este ámbito geográfico tiene como eje principal el río Perené, el cual presenta una longitud de $140 \mathrm{~km}$, se origina en la provincia de Chanchamayo y culmina en la provincia de Satipo. La parte en la que se origina este gran río Perené se considera la parte alta del río, en cuyas márgenes se encuentran las comunidades nativas visitadas.

\subsection{Muestra}

La muestra para el desarrollo del presente estudio está constituida fundamentalmente por la población constituida de adultos bilingües (asháninka-castellano) varones y mujeres; por lo general, grandes conocedores de las historias del pueblo y, además, notables narradores orales. Fundamentalmente las familias de Julia Flores, Carlos Sanchoma, Betty Galarza, Elba Galarza, Enrique Martínez, Carlos Quinchunga y Enrique Casanto Shingari de las comunidades nativas Bajo Chirani y Aldea Bajo Pichanaki. Entre las narraciones que compartieron espontáneamente se encuentran los relatos Nabireri 'Personaje principal', Tasorentsi 'Personaje transformador', Kashiri 'luna', personaje masculino y trascendente en la vida del pueblo asháninka; Manitzi 'tigre', Koshiri 'mono', Kiatsi 'personaje del agua', Pachakamaite 'personaje histórico', Jeto 'araña' y Pakitha 'Gavilán'.

Para el presente estudio, hemos considerado las cláusulas de la narración etnohistórica Pakitha que aún goza de gran vigencia en la memoria colectiva de esta comunidad lingüística y que representa el valor y solidaridad del pueblo asháninka.

\subsection{Trabajo de campo}

Se restableció el contacto con hablantes de las comunidades asháninkas del Perené en el año 2010 para realizar estudios lingüístico-educativos. Dicho contacto tuvo lugar por temporadas breves y se han mantenido hasta la actualidad. 
Avances en la descripción morfosintáctica de la transitividad en el asháninka...

Alicia Alonzo Sutta

Tras el apoyo brindado al presente estudio por parte del Vicerrectorado de Investigación y Posgrado de la UNMSM, se realizó el trabajo de campo a la CN Bajo Chirani, ubicada en la margen izquierda del río Perené, lo que permitió recabar nuevos datos que permitieron corroborar, contrastar e incrementar a los anteriores.

\section{Análisis}

En lo que respecta a nuestro estudio, la transitividad es una propiedad de las cláusulas y no de los verbos. Puesto que en las cláusulas están presentes otros elementos que permitirán dar cuenta de este fenómeno morfosintáctico y semántico. En concordancia con la propuesta de Hopper y Thompson (1980), las cláusulas pueden tener grados de transitividad, estos grados pueden ser más altos o menos altos dependiendo de que en la cláusula se cumplan determinados requerimientos. Si bien es cierto, la llamada hipótesis de la transitividad propuesta por los autores mencionados se basa en el cumplimiento de diez parámetros, en el presente estudio seleccionamos tres, los cuales están expresados a nivel morfosintáctico en las cláusulas de la narración Pakitha.

Los parámetros seleccionados que se expresan en la estructura morfosintáctica de las cláusulas son las siguientes: la presencia del participante 1 expresado a nivel de FN autónoma y/o de prefijo proclítico; la presencia del participante 2 como FN autónoma y/o como sufijo enclítico; la telicidad marcada mediante sufijo - $a k$, correspondiente al 'aspecto perfectivo' seguido de sufijo - $e$ que expresa el 'estado real' de la cláusula. Finalmente, una oración afirmativa en asháninka, que se comprueba mediante la ausencia de marcas léxicas o gramaticales de negación.

Ejemplo:

(I3) Otsitzi yatsikakeri tyapajaniki

$\begin{array}{lll}\text { Otsitsi } & \text { y-atsik-ak-e-ri } & \text { tyapa-janiki } \\ \text { perro } & \text { 3MA-morder-PFV-Real-3MO } & \text { pollo-DIM }\end{array}$

El perro mordió al pollito

En la oración se encuentran expresados en términos morfosintácticos los siguientes parámetros:

282 Lengua y Sociedad. Revista de Lingüística Teórica y Aplicada 
Avances en la descripción morfosintáctica de la transitividad en el asháninka...

a. La FN otsitzi 'perro' corresponde al participante 1, o sea, corresponde al A.

b. La FN tyapajaniki 'pollito' representa al participante 2, es decir, constituye $\mathrm{O}$.

c. La marca sufijal -ak en el verbo corresponde al 'aspecto perfectivo' e indica que la acción se ha realizado. Este sufijo aspectual, necesariamente, se complementa con la marca del estado de realidad que, en este caso, es - e 'estado real' el cual denota una acción realizada.

d. Se comprueba que la cláusula otsitzi yatsikakeri es afirmativa, pues la acción ha tenido lugar, o sea, se ha realizado. Asimismo, no existe una marca adverbial de negación o de duda que exprese lo contrario a lo indicado.

Habiéndose cumplido en la cláusula tres de los parámetros, se considera que esta tendrá un mayor grado de transitividad frente a otra cláusula que no cumpla con tales rasgos.

\subsection{Análisis de las oraciones}

Para dar inicio al análisis de las oraciones seleccionadas, se debe considerar los diferentes tipos de cláusula en la lengua que responden a la siguiente estructura morfosintáctica:

\section{Cuadro 3. Estructura morfosintáctica}

\begin{tabular}{l|l|c}
\hline A & Agente $=$ verbo $=($ objeto $)$ & $\mathrm{O}$ \\
\hline$($ FN $)$ & $\begin{array}{l}\text { Pronominal }=\text { tema verbal-ak-e }=(\text { ri) },(- \text { ro }),(-n a),(-p i),(-a i) \\
\text { Proclítico }=\text { tema verbal-ak-e }=\text { Enclítico }\end{array}$ & $($ FN $)$ \\
\hline
\end{tabular}

El cuadro indica que una oración puede presentar las FN que cumplan el papel de agente y de objeto, pero que estas pueden ser opcionales. Sin embargo, en el verbo debe expresarse el índice prefijal o pronominal, considerado también como un proclítico; luego al tema verbal ha de seguir el morfema de aspecto perfectivo - ak, seguido de sufijo - e que expresa el estado de realidad. A este finalmente y, de manera opcional puede adjuntarse el sufijo proclítico y/o una FN en calidad de participante objeto. 
Avances en la descripción morfosintáctica de la transitividad en el asháninka...

Alicia Alonzo Sutta

\subsubsection{Oraciones con mayor grado de transitividad}

Teniendo en consideración las características morfosintácticas que deben estar presentes en la cláusula, se identifican en la expresión opiratakari 'ella lo ha criado' los dos participantes: el primero se encuentra representado con la marca prefijal $o$ '3NMA' y el segundo mediante sufijo - ri '3MO', los que ya se encuentran marcados con A (agente) y O (objeto).

(14) Tzimatsi aparoni koya opiratari pakitha, opiratakari

$$
\begin{aligned}
& \text { Tzimats-i Aparoni Koya } \mathrm{o}=\text { pira-t-a-ri } \\
& \text { Haber-REAL una mujer 3NM }=\text { criar-EP- REAL-REL } \\
& \text { pakitha } \quad \mathrm{o}=\text { pira-t-ak-a }=\text { ri } \\
& \text { gavilán } \quad 3 \mathrm{NMA}=\text { criar-EP-PFV-REAL-3MO }
\end{aligned}
$$

Otra característica morfosintáctica se encuentra en la marca aspectual - $a k$, la cual indica que la acción ya fue realizada. Finalmente, se comprueba que esta es una oración enunciativa afirmativa. En consecuencia, la oración presenta una mayor transitividad puesto que cumple con presentar los dos participantes A y $\mathrm{O}$; asimismo, la telicidad se encuentra marcada mediante el sufijo aspectual - $a k$ y es afirmativa puesto que no se muestra ninguna marca ni gramatical ni léxica que indique negación.

Cabe precisar que, a pesar de que la cláusula, presenta varias proposiciones, preferimos elegir la última para describir la transitividad, pues constituye la proposición principal de la cláusula analizada. Los otros predicados que tienen como núcleo a tzimatsi y opiratari 'haber' y 'criar' respectivamente, responden a otras características morfosintácticas que se abordarán en estudios posteriores.

A fin de corroborar el análisis anterior, presentamos la oración, cuyo predicado tiene como núcleo a ibetsikakeri.

(15) Ari ibetsikakeri ibanko iroñaka Pakitha.

Ari $\quad \mathrm{i}=$ betsik-ak-e $=$ ri $\quad \mathrm{i}=$ banko iroñaaka pakitha

ADV $3 \mathrm{MA}=$ construir-PFV-Real $=3 \mathrm{MO} \quad 3 \mathrm{PSS}=$ casa ahora gavilán

'Ahí, ahora gavilán ha construido su casa'.

284 Lengua y Sociedad. Revista de Lingüística Teórica y Aplicada 
En la oración, se comprueba que la FN pakitha constituye el participante 1 que actúa en calidad de A. Además de ello y, en cumplimiento con la regla gramatical de configuración de la palabra verbal, se encuentra el índice prefijal $i$ - que hace referencia al participante 1 , es decir, al agente lo que indica que ambas unidades están correferenciadas. En el extremo opuesto del núcleo, se observa la marca del enclítico -ri correspondiente en este caso a 'el objeto perteneciente al género masculino'; precisamente en la FN ibanko 'su casa' se muestra al objeto con rasgo masculino, puesto que el poseedor de dicho objeto es pakitha 'gavilán' asignado como 'ser masculino'.

Complementariamente, en el núcleo del predicado se observa la marca del aspecto perfectivo - ak seguida de - e 'estado real'; ambos indican que la acción ya se ha realizado. Finalmente, se comprueba que el enunciado ari ibetsikakeri ibanko iroñaaka pakitha constituye una oración afirmativa puesto que en su estructura no presenta ninguna marca gramatical ni lexical que exprese negación o duda.

Por consiguiente, estos tres rasgos morfosintácticos descritos indican que la cláusula se encuentra en una alta escala de transitividad.

\subsubsection{Oraciones con menor grado de transitividad}

Por otro lado, existen oraciones en las que su configuración morfosintáctica muestra una ligera diferencia frente a las anteriores; dicha diferencia las califica como cláusulas con transitividad, pero en menor grado que las anteriores. En primer término, debemos señalar que las hemos agrupado porque presentan un rasgo en común referido al estado de realidad; los tres núcleos del predicado presentan el sufijo - $i$ que indica 'estado real'; es decir, indica que la acción se realiza, pero que el evento no se ha completado aún. En la primera oración, el núcleo ijayetzi yayetzi correspondiente a 'él iba llevar' denota una acción que aún no ha finalizado, según la oración que presentamos:

(16) Pakithara ijayetzi yayetzi tsika ipaita atziripayera

$\begin{array}{lll}\text { pakitha-ra } & \mathrm{i}=\text { ja-ye-tz-i } & \mathrm{y}=\mathrm{a}-\mathrm{ye}-\mathrm{tz}-\mathrm{i} \\ \text { gavilán-DEM } & \text { 3MA=ir-PL-EP-REAL } & \text { 3MA=traer-PL-EP-REAL } \\ \text { tsika } & \text { i-pai-t-a } & \text { atziri-paye-ra } \\ \text { PRON } & \text { 3MA=llamar-EP- REAL } & \text { gente-PL-DEM }\end{array}$

'Ese gavilán iba (a) llevar a esos que se llama gentes. 
Avances en la descripción morfosintáctica de la transitividad en el asháninka...

Alicia Alonzo Sutta

En la oración que sigue a continuación, el núcleo iñiiri correspondiente a 'él ve' expresa una acción que no ha concluido.

(17) Ikemeta iñiiri tziyatakotachari atsiri tzibi katonko

$\begin{array}{lll}\text { Ikemeta } & \mathrm{i}=\tilde{n} \text {-ii-ri } & \text { tziyat-ako-t-ach-a-ri } \\ \text { Así } & \text { 3MA=ver-REAL=3MO } & \text { surcar-DAT-EP-PROG-R-REL } \\ \text { atsiri } & \text { tzibi } & \text { katonko } \\ \text { gente } & \text { sal } & \text { río arriba }\end{array}$

'Así él ve a la gente la que surca (río arriba) por sal'

Desde el punto de vista morfosintáctico, esta presenta el sufijo - $i$ que expresa el 'estado real'. Ciertamente, el estado real indica que una acción o un evento sí se realiza. Es indudable que, en ambos casos, la acción se produce; sin embargo, no se concluye. Como se ha observado en las oraciones 1 y 2, una acción se da por concluida siempre que en ella esté presente el sufijo de aspecto - $a k$. La ausencia de este rasgo en las cláusulas tiene una repercusión en la escala de transitividad, según Hopper y Thompson (1980).

De otra parte, respecto a la oración Pakitha tzimatsi pashini iraniri ipaita Kobakitzi se observa que el núcleo del predicado es tzimatsi 'tener' se antepone la FN Pakitha y se pospone otra FN cuyo núcleo es iraniri. Estas FN actúan en calidad de participantes agente y objeto respectivamente.

(18) Pakitha tzimatsi pashini iraniri ipaita Kobakitzi.

$\begin{array}{llll}\begin{array}{l}\text { Pakitha } \\ \text { Gavilán }\end{array} & \begin{array}{l}\text { tzima-ts-i } \\ \text { tener-EP-REA }\end{array} & \begin{array}{l}\text { pashini } \\ \text { DET }\end{array} & \begin{array}{l}\text { ir=aniri } \\ \text { 3M=cuñado }\end{array} \\ \begin{array}{l}\text { i=pai-t-a } \\ \text { 3M=llamar-EP-Real }\end{array} & \text { Kobakitzi } & & \end{array}$

'Gavilán tiene otro cuñado llamado Kobakitzi'.

También hay que anotar que el núcleo del predicado tzimatsi 'él tiene’ muestra una diferencia con respecto a la gran mayoría de verbos en asháninka, pues este no presenta un índice pronominal; es decir, este verbo prescinde de la marca prefijal i-; pero a pesar de ello, se comprueba que el agente de la acción es pakitha 'gavilán'. Lo que destaca en esta unidad es el sufijo - $i$ que expresa el estado real y, que al igual que las oraciones 3 y 4, denota una acción no concluida, lo que implica que 
Avances en la descripción morfosintáctica de la transitividad en el asháninka...

la oración no cumple con el parámetro referido a la telicidad. Para que se cumpla tal requisito, se adjunta al verbo, el aspecto perfectivo - ak que denota en forma contundente una acción concluida.

Ciertamente, se considera que las tres oraciones se encuentran dentro del rango de transitividad, sin embargo, en menor grado que aquellas que presentan el sufijo aspectual -ak que claramente denota una acción concluida y que no está presente en iñiiri. ijayetzi y tzimatsi.

\subsubsection{Oraciones con mínimo grado de transitividad}

Las dos oraciones que aparecen a continuación tienen algo en común que es importante destacar.

(19) ikanta irotaintsi inthonkeri atziri irayeri iroyari.

$\begin{array}{lll}\text { ikanta } & \text { irotaintsi } & \mathrm{i}=\mathrm{n} \text {-thonk-e=ri } \\ \mathrm{ADV} & \mathrm{ADV} & 3 \mathrm{MA}=\mathrm{IRR}-\text { terminar-IRR=3MO } \\ \text { atziri } & \mathrm{ir}=\text { ay-e-ri } & \mathrm{ir}=\mathrm{oy}-\mathrm{a}-\mathrm{ri} \\ \text { gente } & 3 \mathrm{MA}=\text { sacar-IR R-3MO } & 3 \mathrm{MA}=\text { comer-REAL }=3 \mathrm{MO}\end{array}$

'después ya lo saca, lo come, lo terminará la gente'.

(20) iroñaka thame ankithateri antziyatakoteri

iroñaka thame

ADV vamos

a=n-kitha-t-e-ri a=n-tziya-t-ako-t-e-ri

$1 \mathrm{PL}=\mathrm{IRR}$-vestir-EP-IRR=3MO $\quad 1 \mathrm{PL}=\mathrm{IRR}$-surcar-EP-DAT-EP-IRR=3MO

'Ahora vamos a vestirlo, lo haremos surcar'.

En los predicados, se muestran los núcleos verbales inthonkeri y ankithateri que corresponden a 'lo terminará' y 'lo vestiremos'. De donde thonk- y kith-corresponden a los temas verbales 'terminar' y 'vestir'. Ambos se encuentran marcados en los extremos por el morfema discontinuo $n$ - -e, que corresponde al 'estado irreal', estado que denota una acción que aún no se inicia.

Por otra parte, en los predicados de las oraciones en cuestión, se encuentran las marcas de los pronominales $i$ - y $a$-correspondientes al argumento agente (A); así como también, en ambos casos, se encuentra la marca del enclítico 
Avances en la descripción morfosintáctica de la transitividad en el asháninka...

Alicia Alonzo Sutta

-ri correspondiente al argumento objeto $(\mathrm{O})$, dando cumplimiento al primer parámetro.

De acuerdo con la propuesta de Hopper y Thompson (1980), es importante que se cumpla el parámetro referido a la acción télica, es decir, acción realizada. En la medida que este parámetro no se cumple, las dos oraciones analizadas tendrían un menor grado de transitividad en relación con las oraciones de 1, 2, 3, 4 y 5.

Asimismo, cabe indicar que en tanto las oraciones se encuentran expresadas en el estado irrealis, o sea, de acción no realizada, es muy poco probable que en el predicado se registre el morfema de aspecto perfectivo - ak.

A pesar de que las oraciones cumplen con el primer parámetro referente al número de participantes, se debe considerar a las oraciones con un menor grado de transitividad por corresponder a una oración atélica.

\section{Conclusiones}

A partir de lo expuesto, se infiere claramente que la transitividad constituye una propiedad de las oraciones de una lengua y no de los verbos únicamente. Dentro de este marco, se comprueba que en asháninka las unidades argumentales de la oración A y $\mathrm{O}$ son piezas imprescindibles en toda cláusula; estas pueden estar representadas por unidades léxicas y/o unidades gramaticales en calidad de clíticos.

Teniendo en cuenta la afirmación anterior, es preferible considerar a la transitividad como un continuum y evitar la división tajante de verbos transitivos e intransitivos. Dentro de este contexto, consideramos que existen oraciones con menor o mayor grado de transitividad, tal como lo comprobamos en las oraciones presentadas.

Las características morfosintácticas que configuran una oración con mayor grado de transitividad deben presentar unidades léxicas y/o gramaticales que representan a los argumentos centrales de agente $(\mathrm{A})$ y objeto $(\mathrm{O})$. Asimismo, esta debe expresar telicidad mediante la marca aspectual - $a k$. Complementariamente, se debe probar que la cláusula es afirmativa si carece de marcas de negación léxica o gramatical. Cumplidas estas características morfosintácticas en la cláusula, se alcanza un alto grado de transitividad. 


\section{Agradecimiento}

Un agradecimiento especial a las familias asháninkas de las comunidades del Alto Perené quienes me brindaron muy amablemente y con gran sabiduría la historia Pakitha.

\section{Financiamiento}

Este artículo se elaboró en el marco del proyecto de investigación «Análisis morfosintáctico de la transitividad en oraciones de un relato de la lengua asháninka» auspiciada por el Vicerrectorado de Investigación y Posgrado de la Universidad Nacional Mayor de San Marcos en el año 2019.

\section{Conflicto de interés}

Los autores no presentan conflicto de intereses.

\section{Referencias bibliográficas}

Aikhenvald, A. (2000). Transitivity in Tariana. En R. Dixon y A. Aikhenvald (Eds.), Changing Valency case studies in transitivity. Cambridge University Press.

Alonzo, A. (2013). Sustantivos y verbos en el relato asháninka Konoya ipoña Shempiri. Lengua y Sociedad, 13(1), 169-177.

Alonzo, A. (2015). Estructura de la palabra verbal en asháninka. [Estudio inédito realizado con el auspicio del VRIP-UNMSM].

Alonzo, A. (2016). Verbos transitivos en asháninka. [Estudio inédito realizado con el auspicio del VRIP-UNMSM].

Amich, J. (1975). Historia de las misiones del convento de Santa Rosa de Ocopa. Editorial Milla Batres OMF.

Campbell, L. y Grondona. V. (Eds.). (2012). The indigenous language of South America. A comprehensive guide. De Gruyter Mouton.

Casanto, E. (2008). Poñaantsi: Relatos sobre el origen ashaninca. Proyecto Pueblos Indígenas y Desarrollo Sostenible.

Castillo, A. (2017). Aspectos de la frase nominal en caquinte (campa-arawak). [Tesis de licenciatura, Universidad Nacional Mayor de San Marcos]. Repositorio de la Universidad Nacional Mayor de San Marcos.

Castillo, A. (2019). Comandos en Kakinte (Kampa-Arawak). Revista Liames Campinas, 19(1), 1-16.

Corbera, Á. (1994). Fonología e gramática do Aguaruna (Jívaro). [Tesis de doctorado, Universidad Estadual de Campinas-Brasil]. Repositorio 
Avances en la descripción morfosintáctica de la transitividad en el asháninka... Alicia Alonzo Sutta

electrónico de la Universidad de Chile. http://repositorioslatinoamericanos.uchile. $\mathrm{cl} /$ handle/2250/1325236.

Corbera, Á. (2017). Términos de partes del cuerpo humano en mehinacu (Arawak). Lingüística, 33(2), 55-68. http://www.scielo.edu.uy/pdf/ling/v33n2/2079312X-ling-33-02-00055.pdf.

Comrie, B. (1989). Universales del lenguaje y tipología lingüística. Gráficas Cóndor S.A.

Dryer, M. y Haspelmath, M. (Eds.). (2015). The world atlas of language structure. Oxford University Press.

Dixon, R. y Aikhenvald, A. (1999). The Amazonian Languages. Cambridge University Press.

Dixon, R. y Aikhenvald, A. (Eds.). (2000). Changing Valency case studies in transitivity. Cambridge University Press.

Escuela Registral y RENIEC. (2019). Tesoro de nombres asháninkas. RENIEC.

García, F., Mogollón, M., Vallejos, R. y Barraza Y. (2007). Fonología del asháninka del río Pichis. Fonologías, 35-57. FORMABIAP, AIDESEP, ISPP de Loreto, Iquitos.

Givón, T. (2001). Syntax. (Volumen I). John Benjamins Publishing Company.

Gómez, E. (1991). Presente y futuro de las lenguas indígenas. Cátedra Jorge Eliécer Gaitán.

Haspelmath, M. y Sims, A. (2010). Understanding morphology (2. ${ }^{\mathrm{a}}$ ed.). Hodder Education and Hachette, uk Company.

Hopper, P. y Thompson, S. (1980). Transitivity in grammar and discourse. Language, 56(1), 251-299.

INEI. (2007). Junín. Directorio de Centros Poblados y población dispersa. Censos Nacionales XI de Población y vi de Vivienda.

Jacinto, P. y Bernales, D. (2007). Sankinarentsi. СіAмв.

Jacinto, P. (2010). El autorreconocimiento de la identidad: Asháninka vs Ashéninka. Lengua y Sociedad, 10 (1), 67-78.

Kindberg, L. (2008). Diccionario Asháninca. ILv.

Kindberg W. (1975). Formas vacías en el campa asháninca. En Datos Etnolingüísticos.

Lawrence, A. (2013). Inflectional Verbal Morphology in Nomatsigenga. [Tesis de maestría, University of Texas at Austin].

Leclerc, Charles. (1890). Arte de la lengua de los indios antis o campas. Biblioteca Americana. https://archive.org/details/artedelalenguado1adamgoog

290 Lengua y Sociedad. Revista de Lingüística Teórica y Aplicada 
Avances en la descripción morfosintáctica de la transitividad en el asháninka...

Alicia Alonzo Sutta

Malvestiti, M. y Dreidemie, P. (2014). Los verbos genéricos o pro-verbos en la lengua baure (familia Arawak). III Encuentro de Lenguas Indígenas Americanas (ELIA), Libro de Actas, 197-209.

Michael, L. (2006). La incorporación nominal y los clasificadores verbales en el idioma Nanti (Kampa, Arawak, Perú). Proceedings of the Conference on Indigenous Languages of Latin America - II.

Michael, L. (2008). Nanti evidential practice: Language, Knowledge, and social action in an Amazonian society. [Tesis de doctorado, Universidad de Texas]. http:// hdl.handle.net/2152/3884.

Mihas, E. (2010). Essentials of Ashéninka Perené Grammar. [Tesis de doctorado, Universidad de Wisconsin]. http://etnolinguistica.wdfiles.com/local--files/ tese\%3Amihas-2010/mihas_2010.pdf.

Minedu. (2013). Documento Nacional de Lenguas Originarias del Perú. ( $1^{\circ}$ ed.). MINEDU.

Minedu. (2018). Lenguas originarias del Perú. (1ed.). MINEdu.

Moreno Cabrera, J. (1991). Curso universitario de lingüística general I: teoría de la gramática y sintaxis general. Síntesis.

Nichols, J. (1986). Head-Marking and Dependent-Marking Grammar. Language, 62(1), 56-119.

Payne, D. (1991). A Classification of Maipuran (Arawakan) Languages Based on Shared Lexical Retentions. En Handbook Amazonian Languages (pp. 355-499). Desmond Derbyshire.

Payne, D., Payne, J. y Sánchez J. (2008). Morfología, fonología y fonética del Ashéninca del Apurucayali (Campa Arawak preandino) (2a ed.). ILV.

Payne, D. (2008). Diccionario Asheninca-Castellano. ILv.

Payne, J. (2008). Lecciones para el aprendizaje del idioma ashéninca. (2. $\left.{ }^{\circ} \mathrm{ed}.\right)$. ILV.

Payne, T. (1997). Describing morphosyntax: a guide for field linguists. Cambridge University Press.

Pío Aza, J. (2005). Estudio sobre la lengua machiguenga. Ediciones Eunate S.A.

Snell, B. (1998). Diccionario Matsigenka-Castellano. ILV.

Solís, G. (2003). Lenguas en la Amazonía peruana. Programa FORTE - PE.

Swift, K. (1988). Morfología del caquinte. Ministerio de Educación-ILv.

Tallerman, M. (2015). Understanding Syntax (4. ${ }^{\circ}$ ed.). Routledge.

Tsunoda, T. (1999). Transitivity and Intransitivity. Journal of Asian and African Studies, 57, 1-9. 
Avances en la descripción morfosintáctica de la transitividad en el asháninka...

Alicia Alonzo Sutta

Valenzuela, P. (1997). Basic verb types and argument structures in shipibo-conibo. [Tesis de maestría, University of Oregon]. http://citeseerx.ist.psu.edu/viewdoc/ download?doi=10.1.1.864.6412\&rep=rep1\&type $=$ pdf

Vílchez, E. (1996). Fonología del asháninka del gran pajonal con especial atención a los segmentos sibilantes. [Tesis de maestría, Universidad Nacional Mayor de San Marcos]. Repositorio de la Universidad Nacional Mayor de San Marcos.

Weiss, Gerald. (1975). Campa Cosmology: The world of a Forest Tribe in South America. Anthropological Papers of the American Museum of Natural History. http://hdl.handle.net/2246/278

\section{Anexo}

\section{Abreviaturas empleadas}

$\begin{array}{llll}1 & \text { Primera persona } & \text { NM } & \text { No masculino } \\ 2 & \text { Segunda persona } & \text { O } & \text { Objeto } \\ 3 & \text { Tercera persona } & \text { PFV } & \text { A. perfectivo } \\ \text { A } & \text { Agente } & \text { PL } & \text { Plural } \\ \text { ADV } & \text { Adverbio } & \text { PROG } & \text { Progresivo } \\ \text { DAT } & \text { Dativo } & \text { PRON } & \text { Pronombre } \\ \text { DEM } & \text { Demostrativo } & \text { PSS } & \text { Posesivo } \\ \text { DIM } & \text { Diminutivo } & \text { REAL } & \text { Estado realis } \\ \text { DIR } & \text { Direccional } & \text { REL } & \text { Relativo } \\ \text { EP } & \text { Epéntesis } & \text { S } & \text { Sujeto } \\ \text { IRR } & \text { Estado irrealis } & \text { T } & \text { Tiempo } \\ \text { LOC } & \text { Locativo } & \text { FAC } & \text { Tiempo factual } \\ \text { M } & \text { Género masculino } & \text { F } & \text { Femenino } \\ \text { N } & \text { Nombre } & \text { NF } & \text { No futuro }\end{array}$




\section{Trayectoria académica de los autores}

Docente de la Escuela Profesional de Lingüística de la Facultad de Letras y Ciencias Humanas de la UNMSM. Investigadora del Instituto de Investigación de Lingüística Aplicada-Cila. Responsable de los cursos de Gramática de una lengua amazónica I. Realiza investigaciones en la lengua asháninka. 